\title{
Investigation of Steam Condensation Process on Rotating Disk Condenser at Different Rotation Speed
}

\author{
Ali A-M Alasadi, Ph.D \\ Department of Mechanical \\ Engineering \\ University of Baghdad
}

\author{
Akram W. Ezzat, Ph.D \\ Department of Mechanical \\ Engineering \\ University of Baghdad
}

\author{
Ayser Muneer \\ Department of Mechanical \\ Engineering \\ University of Baghdad
}

\begin{abstract}
Theoretical and experimental study has been conducted on steam condensation process on rotating disk condenser at different rotation speed and power supplied to the evaporation tank. The experimental investigation involved, special test apparatus designed and fabricated to realize the condensation process of the saturated steam on the upper surface of a disk whose base is kept at low temperature. The designed system ensures low temperature for the base of the disk equals to $5^{\circ} \mathrm{C}$. The heating element of the steam production sub-system is capable to work at different heating capacities $(385,270,168$ watt). The rotating system is capable to rotate the disc at different rotation speed ranging (103 -150 rpm). Proper slipring is fixed on this shaft to transfer the temperature signals from rotating disc to the read out device. The slip ring is used of 6-way mercury type. The experimental results showed that maximum condensate rate is ensured at rotation speed equals $(\mathrm{N}=150 \mathrm{rpm})$, steam production power supply $\left(\mathrm{P}_{\text {steam }}=385 \mathrm{watt}\right)$ at cooling water temperature $\left(\mathrm{T}_{\text {cool }}=5^{\circ} \mathrm{C}\right)$. It is observed that there is a gradual increase in condensate rate with increasing rotating speed. It is concluded that rotational speed increase leads to increase in the average heat flux across the disc, while local heat transfer is affected by the condensate layer thickness over the upper disc surface. The disk surface temperature measured values decrease continuously in the radial direction and disc average temperature is proportional with its rotational speed as maximum average temperature is reached when this speed equals $150 \mathrm{rpm}$. The film thickness gradually increases from center to outer portion of the disk based on both condensate rate and centrifugal force that affects this thickness which in turn depend on the speed, cooling temperature, water evaporation rate. The agreement between the experimental and theoretical overall heat transfer coefficient appears to be reliable with a deviation of about $(0.7-22 \%)$.
\end{abstract}

\section{Keywords}

Film condensation, Rotating disc, Steam, Different water evaporation power, Theoretical and experimental study

\section{INTRODUCTION}

The recent research contributes to the individual process operations field such as chemical and biochemical reactions, together with heat and mass transfer are extremely important. It is now well recognized that centrifugal forces generated in a rotating system may be used to replace the gravity force in a condensation process. Rotating condensation attractive for space vehicle applications, where the force of gravity is essentially zero. In addition, rotating condensation also seems promising for earthbound applications where higher condensation rates are desired than are obtainable with gravity Sparrow and Hartnett 1961[1]. Since rotating condensation radiators are of great interest in space applications, a condensation experimental has been developed that reduces the effect of gravity. In the ground-based experiments the effect of the gravitational field could be minimized by using a flat plate in which the axis of rotation is parallel to the gravity vector.

Han 1970[2] has presented a detailed review on the present stat of the art with reference to all important prior publications. Heat transfer and fluid flow in thin liquid films with rotation, is of particular interest in several mechanical engineering and chemical processing applications. Thin liquid films on rotating surfaces are encountered in the areas of cryogenics, tempering of glass, drying of textile and paper, transpiration cooling and microgravity thermal fluid handling systems. A number of traditional industries have also turned towards the use of rotating disc compact heat exchangers including chemical process industry, power plants and food processing industry Muhammad et al. [3]. This information together with design procedures for calculating thermal performance will enable an economic assessment of this type of device to be carried out successfully. In many cases attributes other than economic performance may prove to be the most important Khan 1986 [4].

A number of studies related to various aspects of film flow on rotating surface are reported in the literature. These range from the complex flow regimes which were noted above, to the techniques and difficulties associated with the measurement of film parameters such as film thickness and surface velocity. The main area of interest of the present research concerns heat transfer with phase change in thin liquid films. The literature reviewed in this chapter is limited to those papers which make a significant contribution in this area.

Sparrow and Gregg [5] studied analytically film condensation on a rotating disk situated in a large body of pure saturated vapor. The centrifugal filed associated with the rotation of the plate sweeps the condensate outward along the disc surface, and gravity forces are not involved. The problem is formulated as an exact solution of complete Navier-Stokes and energy equations. Numerical solutions are obtained for Prandtl number between 0.003 and 100 for $\mathrm{Cp} \Delta \mathrm{T} / \mathrm{hfg}$ in the range of 0.0001-1.0. Sparrow and Gregg [6], in a technical brief in February 1960, added the effect of vapor drag to their previous analysis of heat transfer and showed that the effect was quit small. It was claimed that the deviation between theory between theory and experimental must be attributed to other causes. Nandapurkar and Beatty [7] their experimental results using methanol, ethanol, and Freon-113 at atmospheric pressure in. The use of centrifugal force has been shown to be effective for the continuous removal of condensate from a horizontal plate condenser. Hoyle and Matthews [8] studied the heat transfer from steam to cooled rotating cylinder, in which drainage of the film is perpendicular to the surface by the growth and throwing off of protrusions. Espig, 1964 carried out a series of experiments to study the transfer of heat from steam to a cooled vertical flat rotating disc. In these experiments the drainage of the film was by a flow along the 
surface of the disc. Homam et. al [9] studied experimentally heat transfer parameters of film condensation on a rotating flat plate. A rotating flat-plate condensation experiment has been developed to investigate the heat transfer characteristics of a rotating flat-plate radiator. The condensing surface is cooled to simulate the rejection of heat to a cold surface. The working fluid is superheated steam. The results obtained including relationship between the overall heat transfer coefficient and the temperature difference between the working fluid and a cold environment, both placed in dimensionless groups, and plate angular rotational speeds. This empirical relationship is useful for choosing the optimum rotational speed for a rotating flat-plate radiator given a desired heat rejection load. This study also provides the basis for designing new heat rejection systems using centrifugal forces and condensation phenomena in both space and ground applications. Yanniotis and Kolokotsa [10] studied the condensation of saturated low pressure water vapor on the bottom side of a smooth surface horizontal aluminum spinning disc experimentally. Results of the heat flux on the disc as a function of the wall superheat and the speed of rotation show that the mean heat transfer coefficient varies between $10 \mathrm{~kW} / \mathrm{m} 2 \mathrm{k}-20 \mathrm{~kW} / \mathrm{m} 2 \mathrm{k}$ during rotation speed $(0-$ $1000 \mathrm{rpm})$ and high heat flux rang.

Muhammad et. al [3], worked experimentally and theoretically by which he described hydrothermal characteristics of liquid flowing on horizontal rotating disk. The liquid was introduced at the center of the disk in the form of a falling jet. Liquid spread out due to the simultaneous action of surface and body forces and forms a thin film over the disk. The effect of nozzle to plate spacing, fluid flow rate and disk rotational speed on the film thickness, local heat transfer coefficient and local Nusselt number along the radial direction was investigated. It was observed that local heat transfer coefficient and local Nusselt number strongly depends upon flow rate and disk rotational speed and has no influence of nozzle to plate spacing. The study also concludes that the process of heat transfer is intensified manifold by rotating the surface, which will be helpful in the future heat and mass transfer applications.

The objectives of the present work are to investigate experimentally and theoretically the steam condensation process on rotating disc condenser at different rotation speed, and water evaporation power which is related to steam production rate. Steam condensation process is evaluated and measured based on heat transfer between the condensed liquid flowing over the surface of rotating disc and coolant fluid used for disc cooling purpose. The main objectives of the present work are:

1. Investigate the relation between the condensation rate versus disc rotating speed experimentally and theoretically.

2. Investigate the relation between water condensate thickness versus disc radius affected by both condensate rate and centrifugal force of disc rotation.

3. Investigate the effect of heat transfer coefficients on the condensation process.

Figure (1) and Figure (2) shows the elementary principles of the condensation process on rotating disc and the different parameters effects on this process.

\section{EXPERIMENTAL WORKE}

The test rig consists of rotating disc shown figure (3), which has a diameter of $150 \mathrm{~mm}$ and thickness of $2 \mathrm{~mm}$ is made from high-strength, high conductivity stainless steel alloy, this assembly rotates about a hollow shaft using proper ball bearing. The rotating shaft is of $450 \mathrm{~mm}$ length and is bored out to $18 \mathrm{~mm}$, while the outer diameter is machined to suit the ball bearing internal diameter. Two pulleys of $200 \mathrm{~mm}$ and $250 \mathrm{~mm}$ diameter are made from aluminum and fixed to the rotating shaft by screw. The two pulleys and their accessories that represent disc rotating mechanism is shown in figure (4). The upper driving shaft is clamped on the small shaft, which has a diameter of $20 \mathrm{~mm}$ and $120 \mathrm{~mm}$ length. It is made from high-strength stainless steel alloy and screwed to the slip-ring.

The driving pulley is mounted below this housing on the shaft. Both pulleys (driving and driven) are made of Aluminum with different diameters, i.e. $200 \mathrm{~mm}$ for the first and $57 \mathrm{~mm}$ for the other. A $37 \mathrm{KW}$ D.C. motor (3 Phase, type JL 712-4B3) is provided to the main drive. The variable speed rotation is achieved by a speed controller system (type SVIC5 Manual). With this arrangement the rotation speeds of $103,113,124,135,140$ and $150 \mathrm{rpm}$ are realized, knowing that maximum speed of the motor is $2000 \mathrm{rpm}$. In view of this problem, the local temperature gradient normal to the disc surface was obtained by smoothing the temperature output from six thermocouples pairs (types K) are placed in $(24,32$, $39,46,53$, and $60 \mathrm{~mm}$ ) distances from the disc center for the purpose of measuring disc upper surface temperature see figure (5). The ends of thermocouples wires are contacted to slip-ring. The slip- ring is connected to the board of the data logger through the upper end of the shaft. All thermocouples are calibrated in place according to the temperature range that is used during the experiments. The liquid film temperature is measured with a thermistor which had previously been calibrated using a high precision chromium-nickel thermometer $\left( \pm 10-3^{\circ} \mathrm{C}\right)$. The accuracy of the thermocouples is $\pm 0.5^{\circ} \mathrm{C}$. The bolted gasket is fixed by the silicon rubber gasket on the up end cylinder from outer periphery for the purpose of preventing steam leakage to the cooling water vessel. The collector dish is fixed on the cooling vessel surface by $6 \mathrm{~mm}$ diameter fixtures to ensure uniform water distribution on this surface.

Some auxiliary equipment's in the experimental set-up are used during test rig operation and conducting the related experiments. These are: centrifugal pump to deliver the cold water for rotating disc cooling purpose, stop watch, two thermometers one used to measure temperature of the weather in the laboratory while the other is used to measure the temperature of water in steam condensate collector dish, laboratory flask (laboratory glass blowing).

Saturated water vapour at $45-60^{\circ} \mathrm{C}$. The steam is feed continuously towards the rotating disc via 6 nozzle ports which provides the steam on the surface disc, water is fed to the heating tank of 15 liter capacity. The level inside the tank is measured using an $\mathrm{L}$ shaped manometer which is attached to the bottom of the tank. An immersed heater of $1.5 \mathrm{KW}$ power rating is used to heat the water inside the tank. The heater power is regulated to ensure desired water temperature using thermostat. Steam is fed to the test rig using $31 \mathrm{~mm}$ inside diameter and $38 \mathrm{~mm}$ outside diameter pipe. Stainless steel tubes are used for distributing steam via stationary annular feed channel which delivers the steam to the test section via nozzle, distributor. Another feeding channel made of $15 \mathrm{~mm}$ inner diameter and $22 \mathrm{~mm}$ outside diameter rubber tube. The pressure in the steam chamber is controlled by pressure reducing valve. The condensed steam in liquid droplets moves in the radial direction due to the centrifugal 
force and collected in circular rubber jacket and drawn through plastic tube to the collection flask.

The cooling liquid is introduced through, cold water system that is designed to ensure low temperature for the base of the disk. Cold water system consists of, condenser ( $270 \mathrm{~mm}$ x 280 $\mathrm{mm})$, evaporated $(300 \mathrm{~mm} \times 210 \mathrm{~mm})$, expansion valve and compressor (capacity $1 / 6 \mathrm{hp}$ ) to supply the cold water temperature $5^{\circ} \mathrm{C}$ from this system by digital thermostat is pumped and distributed from the center of the discs towards its periphery in a thin film under the action of centrifugal force.

\subsection{Operating and Experimental Procedure}

After installation of the rotor and disc assembly is completed, the rotating unit is balanced to minimize vibrations. A number of tests are performed with water and steam to ensure the sealing of the unit covered rotational speed range of 103 to $150 \mathrm{rpm}$. Following these preliminary tests it is decided to investigate heat transfer to thin liquid films (for sensible heating with subsequent evaporation) for the conditions listed below. Feed flow rate $=200 \mathrm{ml}$, inlet temperature $=100^{\circ} \mathrm{C}$ boiling point, speed of rotating $=103 \mathrm{rpm}$ to $150 \mathrm{rpm}$, temperature of cooling $=5^{\circ} \mathrm{C}$, power supply $=168$ watt to 385 watt. The quantity of the steam condensates procured at the desired speed of rotation is measured. The pump in cooling water system is then switched on and the desired flow rate is attained.

Since the facility is heavily lagged and the residence time and hold up are very small, the condensations in the test section achieved a steady state very rapidly allowing collection of the following data to commence immediately.

a. Heater power, condensate rate and cooling water flow rate.

b.Inlet and outlet temperature of steam, cooling water and condensate.

c. Temperature on the rotating disc surface.

d. Speed of rotation.

e. Power consumed by steam.

\section{ANALYSIS OF LIQUID FILM ON ROTATING DISCS}

Analytical solution for the condensation mechanism on rotating circular cold surface will be modeled using finite difference model. The mathematical model takes under consideration the effect of rotation speed, steam temperature and disk temperature profile on the condensation rate and condensed steam boundary layer growth along its radius.

Following assumptions are taken under consideration during modeling the present case study:

1. Only radial flow of the condensate film is considered indicating that the effect of Coriolis force due to the relative movement of the condensing film on the disc is negligible under the experimental conditions.

2. The following input parameters are based during conducting theoretical approach:

a) Disc thermal conductivity. b) Physical dimension of the disc. c) Heat transfer between the steam and the cooled disc which will be calculated based on proper formulas. d) Speed of rotation which will be identified based on the characteristics of rotating system. e) Physical properties of the condensed steam. f) steam flow rate inside the closed rotation system.

3. The calculations are based on the following assumptions: a) The fluid properties are constant. b) Zero shear stress at the condensate/vapor interface. c) The liquid is Newtonian. d) Liquid is introduced to the disc surface in an axisymmetric manner. e) Incompressible flow. f) Steady state condensate film flow. g) Film surface is smooth.

Vapor is pure and there is no non-condensable gas

The flow of condensate in the film is laminar within rotation speed range that will be adapted during the experiments.

The hydrodynamics of these flows have been accurately described in terms of a simple balance between centrifugal force and the viscous forces in the film IZV [11]:

$$
\vartheta \frac{d u_{r}^{2}}{d y^{2}}=-r \omega^{2}
$$

The radial velocity could be estimated by integrating the equation (3.1) for the conditions of on slip at the disc surface $(\mathrm{y}=0)$ and for stress free $\left(d u_{r} / d y=0\right)$ conditions at $(\mathrm{y}=\delta)$. The redial velocity distribution across the film is found to be parabolic for these conditions:

The average film velocity,

$$
\begin{aligned}
& \overline{u_{r}}=\frac{1}{\delta} \int_{o}^{\delta} u_{r} d y \\
& \overline{u_{r}}=\frac{\omega^{2} r \delta^{2}}{3 v}
\end{aligned}
$$

The estimation of the film thickness as a function of radial

$$
u_{r}=\frac{r \omega^{2} \delta^{2}}{v}\left[\left(\frac{y}{\delta}-\frac{1}{2}\left(\frac{y}{\delta}\right)^{2}\right)\right]
$$
position, rotational speed, flow rate, and rheological properties of the liquid has been based on the equation

The average velocity $\overline{u_{r}}$ is redefined in terms of flow rate per unit area equating with equation (3.3), i.e.

$$
\begin{aligned}
& \overline{u_{r}}=\frac{Q}{2 \pi \delta}=\frac{\omega^{2} r \delta^{2}}{3 v} \\
& \text { or } \delta=\left(\frac{3}{2 \pi} \frac{Q v}{r^{2} \omega^{2}}\right)^{0.333}
\end{aligned}
$$

The Nusselt treatment of condensing heat transfer was based on the assumption that latent heat was conducted from the steam interface at a temperature $\mathrm{T}_{\mathrm{s}}$ to the cooled wall at a temperature $\mathrm{T}_{\mathrm{w}}$. this gives rise to a heat transfer coefficient $h=(K / \delta)$. However, in the present case it is assumed that there is no heat flux across the steam interface as the liquid film approaches the wall temperature. The average mixed film temperature which was measured during the experimental is given by

$$
T_{m}=\int_{0}^{\delta} u T d Z / \int_{0}^{\delta} u d Z
$$

Noting that the parabolic temperature distribution which satisfies the conditions $\mathrm{T}=\mathrm{T}_{\mathrm{w}}$ at $\mathrm{Z}=0, \mathrm{~T}=\mathrm{T}_{\mathrm{s}}$ at $\mathrm{Z}=\delta,(\mathrm{dT} / \mathrm{dy})=$ 0 at $y=\delta$, is 


$$
\left[\left(T-T_{o}\right) /\left(T_{\delta}-T_{o}\right)\right]=\left(\frac{3 Z}{2 \delta}-\frac{1}{2} \frac{Z^{3}}{\delta^{3}}\right)
$$

The velocity profile is used from the Nusselt model. It is derived for the similar conditions of flow i.e. laminar

$$
\frac{u}{u_{\delta}}=\frac{2 Z}{\delta}-\frac{Z^{2}}{\delta^{2}}
$$

Substitution of the values of $\mathrm{T}$ and $\mathrm{u}$ into equation (3.5) from equation (4.6) and (4.7) and then integrating it, leads to

$$
\left(\frac{T-T_{o}}{T_{m}-T_{o}}\right)=\frac{40}{61}\left(\frac{3 Z}{\delta}-\frac{Z^{3}}{\delta^{3}}\right)
$$

or

$$
\left.\left(\frac{d T}{d Z}\right)\right|_{Z=0}=\left(\frac{120}{61 \delta}\right)\left(T_{m}-T o\right)
$$

Rate of heat transfer per unit area can be written as;

$$
q^{\prime}=-\left.K\left(\frac{d T}{d Z}\right)\right|_{Z=0}=h_{r}\left(T_{m}-T_{o}\right)
$$

Where $\left(h_{r}\right)$ is the local value of the heat transfer coefficient at the distance ' $r$ ' from the origin.

Comparison of equation (3.8) and (3.9), led to the following simplified expression, to define local heat transfer coefficient;

$$
h_{c}=\frac{120}{61}\left(\frac{K}{\delta}\right)
$$

Where, $(\delta)$ is the thickness of the liquid film and it could be determined by the equation given in this chapter (defined in section 4.2.3)

$$
\delta=\left(3 Q \mu / 2 \pi \omega^{2} \rho r^{2}\right)^{0.333}
$$

Substituting the value of $(\delta)$ into equation (3.10) and the average value of heat transfer coefficient for the effective heat transfer area is obtained by integrating equation (3.10)

$$
\begin{aligned}
& \bar{h} c=\frac{1}{A} \int_{R_{I}}^{R_{o}} h r d A \\
& \bar{h}_{c}=\frac{90}{61}\left(\frac{r_{o}^{8 / 3}-r_{i}^{8 / 3}}{r_{o}^{2}-r_{i}^{2}}\right)\left[K_{L}\left(\frac{2 \pi \omega 2 \rho}{3 Q \mu}\right)_{L}^{0.333}\right]
\end{aligned}
$$

Liquid side film coefficient is estimated using the following correlation, Butuzov and Rifert [12].

$$
h_{f}=2.18\left(\frac{K_{f}^{3} \rho_{f}^{2} N^{2} r_{o}^{2}}{\omega_{f} \mu_{f}}\right)^{1 / 3}
$$

Condensate rate

$$
Q_{c}=\left(q A / \rho_{c} h_{f g}\right)
$$

Overall heat transfer coefficient

$$
\frac{1}{U}=\frac{1}{h_{c}}+\frac{1}{h_{f}}+\frac{x}{K}
$$

Experimental overall heat transfer coefficient:

$$
U=\frac{q}{\left(T_{s}-T_{\text {sur }}\right)}
$$
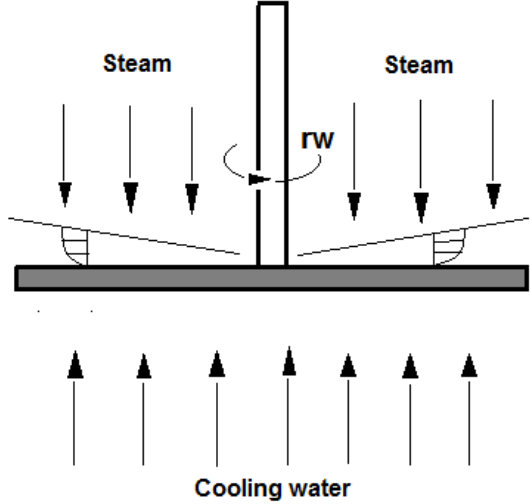

Fig. (1) The Rotating Disc System

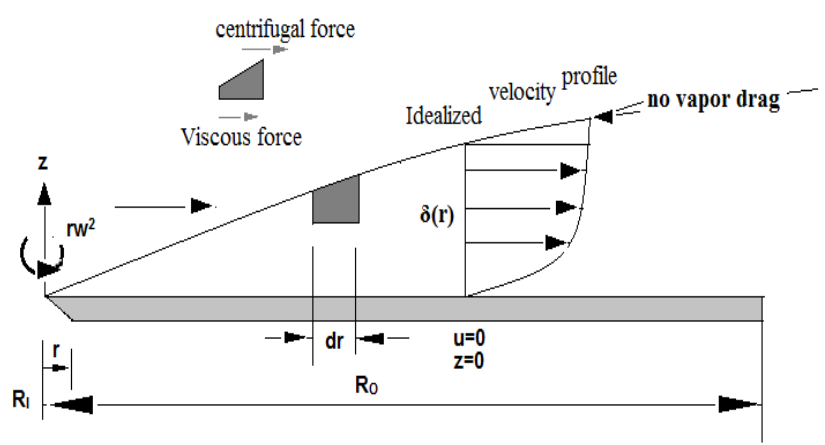

Fig. (2): The Volume Element of Condensate on a Rotating Disc 

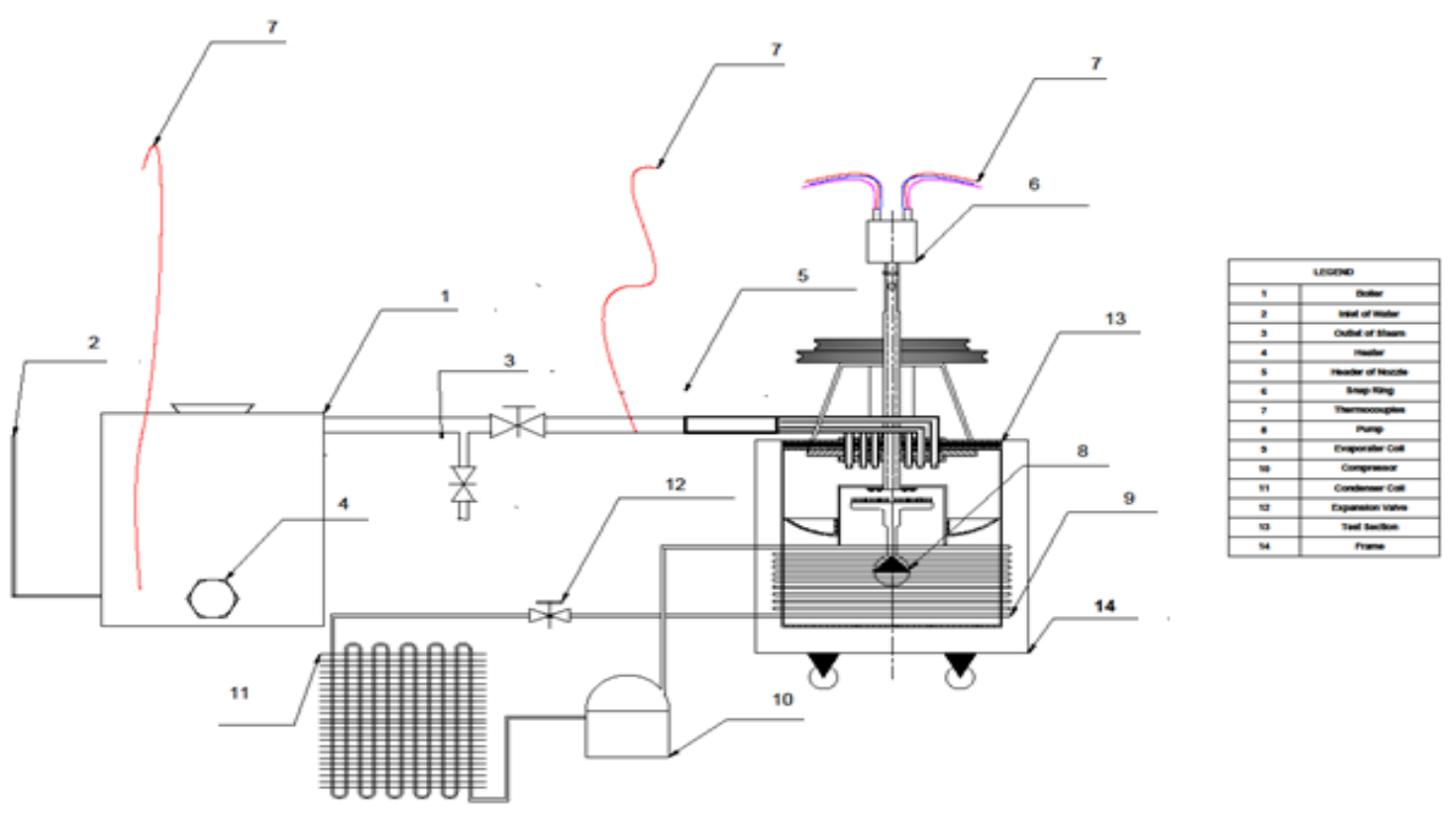

Fig. (3): Condensation test rig lay out

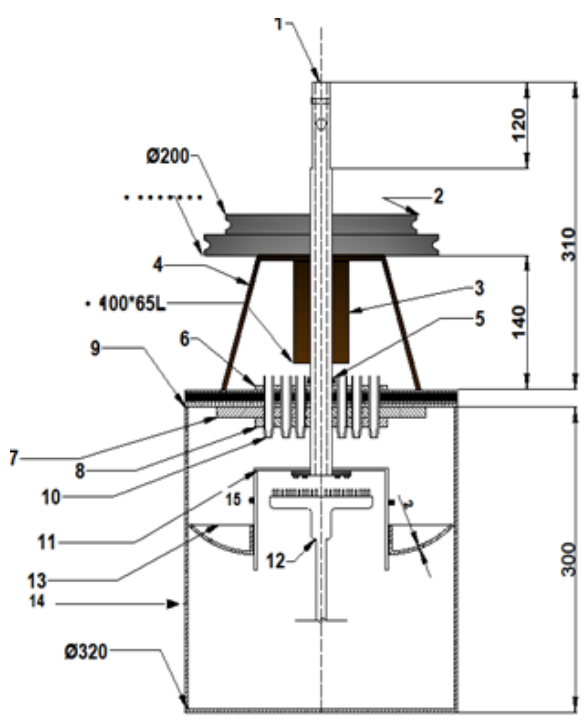

Fig. (4) Rotating System

\begin{tabular}{|c|c|}
\hline \multicolumn{2}{|c|}{ Test Section Detail } \\
\hline 1 & Shaft(s.s) \\
\hline 2 & Pully(AL) \\
\hline 3 & Flang \\
\hline 4 & Support \\
\hline 5 & Ball Bearing \\
\hline 6 & Upper Flang \\
\hline 7 & Middl Flang ø220 \\
\hline 8 & Lower Flang ø130 \\
\hline 9 & Test Section Cover \\
\hline 10 & Nozzle 6numbers \\
\hline 11 & Rotating Disc \\
\hline 12 & $\begin{array}{c}\text { Injection (Nozzle ) Cooling } \\
\text { Water(S.S) }\end{array}$ \\
\hline 13 & $\begin{array}{c}\text { Steam Condensate Collector } \\
\text { Dish(S.S) }\end{array}$ \\
\hline 14 & surface cooling water \\
\hline 15 & gasket \\
\hline
\end{tabular}

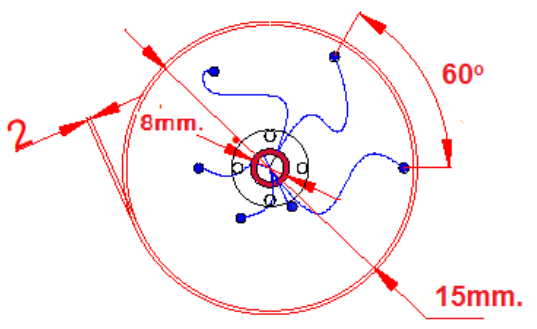

Fig. (5): Rotating disc 


\section{RESULTS AND DISCUSSION}

The effect of the following parameters ranges on the steam condensation rate is investigated in the experimental and theoretical parts:

1. Speed rotating, $\mathrm{N}$ range $(103,115,124,135,140$ and 150) rpm.

2. The power supplied to the evaporation tank, P Range (385, 270 and 168 watt).

\subsection{Steam Condensation Rate}

Figure (6) represents the rate of condensate steam on the surface disc with effect of rotating speed with different power supply at $5^{\circ} \mathrm{C}$. They show that for $\mathrm{P}_{\text {steam }}=385$ watt, the heat transfer on the disc surface have more effect when the speed of rotation increases and vapor condensation rate increase at constant cooling water temperature $\left(\mathrm{T}_{\text {cool }}=5^{\circ} \mathrm{C}\right)$, i.e. vapor condensation rate is proportional to heat transfer rate and inversely proportional with condensate film thickness over the rotating disc. It is concluded that rotational speed increase leads to increase in the average heat flux across the disc, while local heat transfer is affected by the condensate layer thickness over the upper disc surface.

\subsection{Disc surface temperature}

Figures (7) to (9) show that temperature measured values decrease continuously in the radial direction at cooling water temperature equal $5^{\circ} \mathrm{C}$ at different water evaporation power ranging $(385,270$ and 168 watt). From other hand rotating speeds of the rotating disc $(103,115,124,135,140$ and 150 rpm) affect temperature distribution also. This effect could be observed as surface temperature increase proportionally with rotation speed. The reason is that the rotating speed reduces the film thickness along the radius which as it accelerates the fluid and enhances the rate of heat transfer. Disc average temperature is proportional with its rotational speed as maximum average temperature is reached when this speed equals $150 \mathrm{rpm}$. It is also clear that disc surface temperature increases by increasing water evaporation power $(385,270$ and 168 watt) due to both condensate film thinning versus velocity and augmentation of condensate rate versus steam production rate which increase its condensation rate. From other hand any increase in condensate film thickness with the radial distance increase heat transfer resistance which in return decreases the heat flux to fluid in the radial direction and this clear from temperature radial distribution versus disc radius. Measurement results related to the effect of disc rotation speed on the disc redial temperature distribution at constant cooling water $5^{\circ} \mathrm{C}$ and different water evaporation power are shown in figures (10) to (12).

\subsection{Film Thickness}

Figure (13) and (14) describe the effect of rotational speed on film thickness versus disc radius at cooling water temperature $5^{\circ} \mathrm{C}$ and different water evaporation power. It could be seen that film thickness increases versus radial locations, while this thickness is inversely proportional to the rotation speed. This decrease is justified by increasing the centrifugal force effect. However, the effect is more appreciable for high rotational speeds. The film thickness peak location shifts to $\delta=0.565 \mu \mathrm{m}$ when rotational speed $\mathrm{N}=103 \mathrm{rpm}$ and $\mathrm{T}_{\text {cool }}=5^{\circ} \mathrm{C}$. The effect of the rotational speed on the condensate film thickness as discussed before is due to the fact that the surface rotation add extra inertia that influence on the rate of film thickness by decreasing the disc surface temperature at constant rotational speed, due to the large volume of fluid that take more heat away from the system.

\subsection{Overall Heat Transfer Coefficient}

Figure (15) shows the average heat transfer coefficients between of minimum and maximum values of $\mathrm{U}=2.2 \mathrm{E}+03$ and $2.57 \mathrm{E}+03 \mathrm{w} / \mathrm{m}^{2} \mathrm{k}$ respectively at evaporation power supply $=385 \mathrm{w}$ at a constant cooling water $\mathrm{T}_{\text {cool }}=5 \mathrm{C}^{\circ}$, while minimum and maximum values of $\mathrm{U}=2.1 \mathrm{E}+03$ and $2.5 \mathrm{E}+03$ $\mathrm{w} / \mathrm{m}^{2} \mathrm{k}$ respectively at water evaporation power $=270 \mathrm{w}$. These values reaches to $2.05 \mathrm{E}+03$ and $2.4 \mathrm{E}+03 \mathrm{w} / \mathrm{m}^{2} \mathrm{k}$ respectively at evaporation power supply $=168 \mathrm{w}$. It is concluded that overall heat transfer coefficient increases by increasing disc rotation speed due to the film thinning effect caused by centrifugal effects. Figure (16) describe the experimental results related to the effect of varying rotational speed on overall heat transfer coefficient at cooling water temperature $5^{\circ} \mathrm{C}$. Similar behavior to theoretical results is concluded from these figures. However it is noted that experimental overall heat transfer coefficient rate is lower than theoretical values. This difference is estimated to be consistently $(2-25 \%)$ below the predictions of the experimental overall heat transfer coefficient rate depending on disc rotation speed and water evaporation power. Figures (17) to (19) represent this comparison. The agreement between the experimental and theoretical overall heat transfer coefficient appears to be reliable with a deviation of about $(0.7-28 \%)$. This deviation is justified by experimental apparatus accuracy and the assumptions used in the theoretical approach. It is concluded that overall heat transfer coefficient increases by increasing disc rotation speed due to the film thinning effect caused by centrifugal effects. From same figures it is clear that more heat is transferred as the power supplied to the water tank increases. It is concluded that condensate rate is inversely proportional to cooling water temperature.

\section{CONCLUSIONS}

The conclusions obtained from the present investigation shows that surface temperature of the disc increases proportionally with steam production rate at constant disc rotating speed and increases proportionally with increasing disc rotating speed at constant steam production rate. Increase in heat transfer film coefficients are observed at the inner radial positions where the feed steam is approached the disc angular velocity. Steam condensation rate is proportional to disc rotation speed as it increases by increasing rotational speed. According to previous studies for each steam production rate there is certain speed at which the condensate rate is saturated. The speed is not reached during relevant experiments. Based on the heat transfer rate distribution the condensate rate increases with increasing steam - air mixture in the rotating disc chamber due to the increase of steam production rate by keeping cooling water temperature $5^{\circ} \mathrm{C}$. Overall heat transfer coefficient rate is proportional to disc rotational speed as it increases with increasing rotational speeds at cooling water temperature $5^{\circ} \mathrm{C}$. Disc rotation speed and steam production rate have different effects on the condensate film thickness growth versus radial direction as this thickness is affected by both the centrifugal force and the condensate rate at each radius. The condensate film thickness reaches to its maximum value when $P_{\text {steam }}=385 \mathrm{w}, \mathrm{T}_{\text {cool }}=5^{\circ} \mathrm{C}$ and $\mathrm{N}=103 \mathrm{rpm}$ among the whole experimental range. Condensate film thickness inversely affects the heat transferred between disc surface and the surrounding steam. From other hand this heat transfer rate is in return function of the disc surface temperature. It is further noted that the rate of thinning of condensate film strongly the influenced by cooling 
parameter. The maximum of the film thickness rate $\delta_{\max }$ $=.57 \mu \mathrm{m}$ at $\mathrm{N}=103 \mathrm{rpm}, \mathrm{T}_{\text {cool }}=5^{\circ} \mathrm{C}$ and steam production power $=385$ watt. The agreement between the experimental and theoretical overall heat transfer coefficient appears to be good with maximum deviation of about $(8.7-28 \%)$ and minimum deviation of about (0.7-14\%).

\section{NOMENCALTURE}

$\begin{array}{lll}\mathrm{D}, \mathrm{d} & \text { Diameter } & \\ \mathrm{h} & \text { Heat transfer coefficient } & \mathrm{w} / \mathrm{m} 2 \mathrm{k} \\ \mathrm{K} & \text { Thermal Conductivity } & \mathrm{w} / \mathrm{m} . \mathrm{k} \\ \mathrm{N} & \text { Rotational speed } & \mathrm{rpm} \\ \mathrm{Q} & \text { Volumetric flow rate } & \mathrm{m}^{2} / \mathrm{sec} \\ \mathrm{q} & \text { Heat flux } & \mathrm{w} / \mathrm{m}^{2} \\ \mathrm{R}, \mathrm{r} & \text { Radius } & \mathrm{m} \\ \mathrm{T} & \text { Temperature } & { }^{\circ} \mathrm{C}, \mathrm{k} \\ \mathrm{U} & \text { Overall heat transfer coefficient } \mathrm{w} / \mathrm{m}^{2} \mathrm{k}\end{array}$

\section{Greek Letters}

$\delta \quad$ Film thickness

$\lambda$ Latent heat

$\mu \mathrm{m}$

\section{$\mu \quad$ Viscosity of liquid}

$v$ Kinematics viscosity of the liquid

$\rho$ Density

$\tau$ Shear stress

$\omega$ Angular velocity

$\mathrm{Ns} / \mathrm{m}^{2}$

$\mathrm{m}^{2} / \mathrm{s}$

$\mathrm{kg} / \mathrm{m}^{2}$

$\mathrm{N} / \mathrm{m}^{2}$

$\mathrm{rad} / \mathrm{s}$

\section{REFERENCES}

[1] Sparrow, F, M, and Hartnett, J. P. (1961), " Condensation on a rotating Con",Int. J. Heat Transfer, vol. 83, pp. 101107.

[2] Han S. T., Drying of paper (1970), TAPPI 53(6), pp.10341046.

[3] Muhammad S., Khan J. R., Suleman H., Naveed S. (2011), "Hydrothermal Characteristics of Thin Liquid Film Flow on Horizontal Rotating Disc", J. Quality and Technology Management, Vol. Vll, pp. 131-147.

[4] Khan J. R. (1986), "Heat Transfer on a Rotating Surface with and without phase Chang", Ph.D. Thesis, University of Newcastle upon Tyne.

[5] Sparrow. E.M., Gregg, J. I. (1959), "A Theory of Rotating Condensation, J. Heat transfer", Vol. 81, pp. 113-120.

[6] Sparrow. E.M., Gregg, J. I.(1960), "A boundary Layer Treatment of Laminar Film Condensation", A.S.M.E. Paper No. Vol. 58-SA-Z.

[7] Nandapurkar, S. S., and Beatty, K. O. (1960),"Condensation on a Rotating Disc", Chem. Eng. Prog. Symp., Vol. 56(30), pp. 129-137.

[8] Hoyle, R. and Matthews, D. (1965), "The Effect of Speed on the Condensate Layer on a Cold Cylinder Rotating in a Steam atmosphere", Int. J. Heat Mass Transfer, Vol. 22,105 .

[9] Homam M. Al-Baroudi and Andrew C. Klein (1995), "Experimental Simulations and Heat Transfer Parameter Measurements of Film Condensation on a Rotating Flat Plate", Experimental Thermal and Fluid Science, pp. $\underline{124-135 .}$
[10] Yanniotis S. and Kolokotsa (1996)," Experimental Study of Water Vapour Condensation on a Rotating Disc", Int. Comm. Heat Mass Transfer, Vol.23, pp.721-729.

[11] Vachagin, F. D., and Nikolaev, V. S.,(1960),"Flow of a Viscous over the Surface of a Rapidly Rotating Flat Disc", IZV. Khim. Ikhim. Tekh., Vol. 3, pp. 1097-1102.

[12] Butuzov, A.I., and Rifert, V.G., "Heat Transfer in Evaporation of Liquid from a Film on a Rotating Disc" Heat Transfer -Soviet Research, 50(1) (1973) pp 88-94.

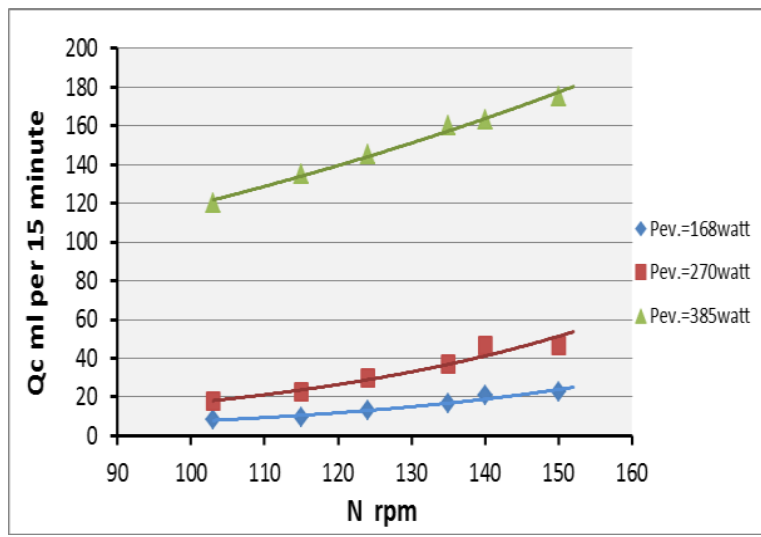

Fig. (6): Effect rotating disc speed on the steam condensate volumetric rate at a disc surface temperature $\left(\mathrm{Ts}=5^{\circ} \mathrm{C}\right)$

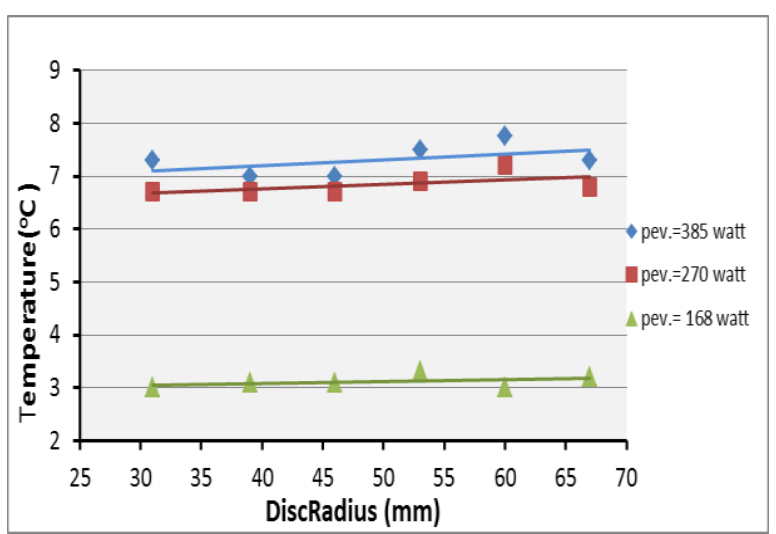

Fig. (7) Disc surface temperature variation versus disc radius at a constant cooling water temperature $\left(T=5^{\circ} \mathrm{C}\right)$ and rotating disc (103 rpm) 


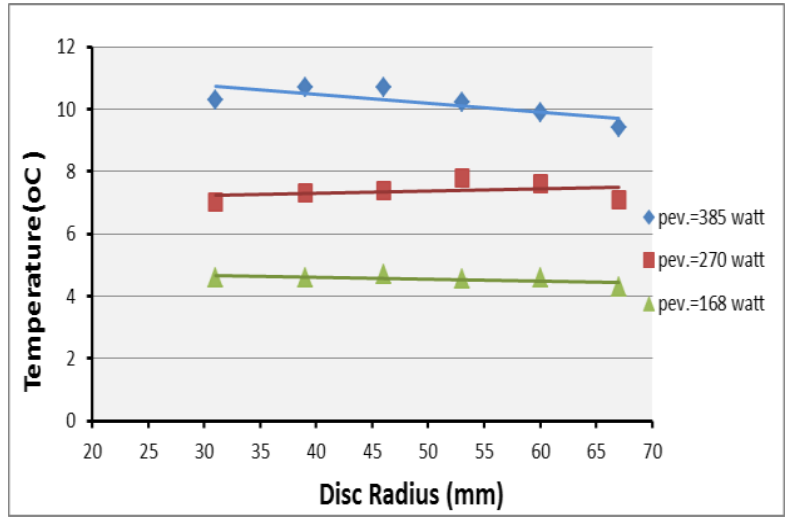

Fig. (8) Disc surface temperature variation versus disc radius at a constant cooling water temperature $\left(T=5^{\circ} \mathrm{C}\right)$ and rotating disc (124 rpm).

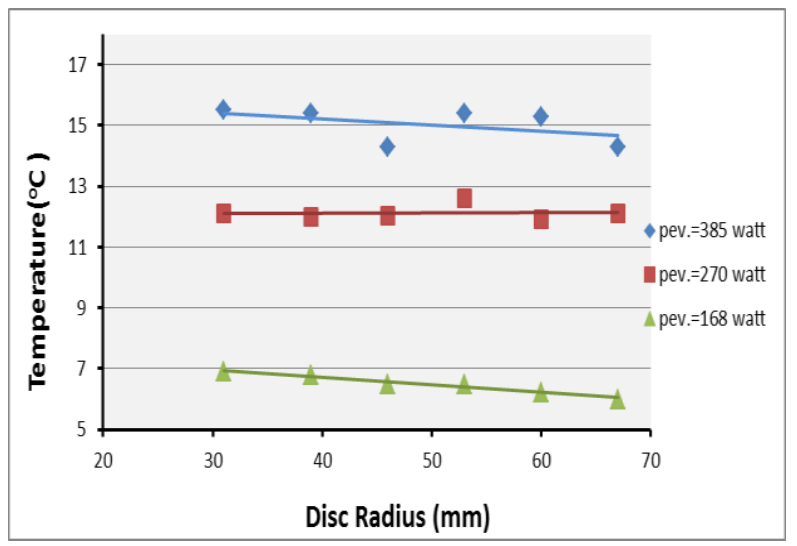

Fig. (9) Disc surface temperature variation versus disc radius at a constant cooling water temperature $\left(T=5^{\circ} \mathrm{C}\right)$ and rotating disc (150 $\mathrm{rpm})$

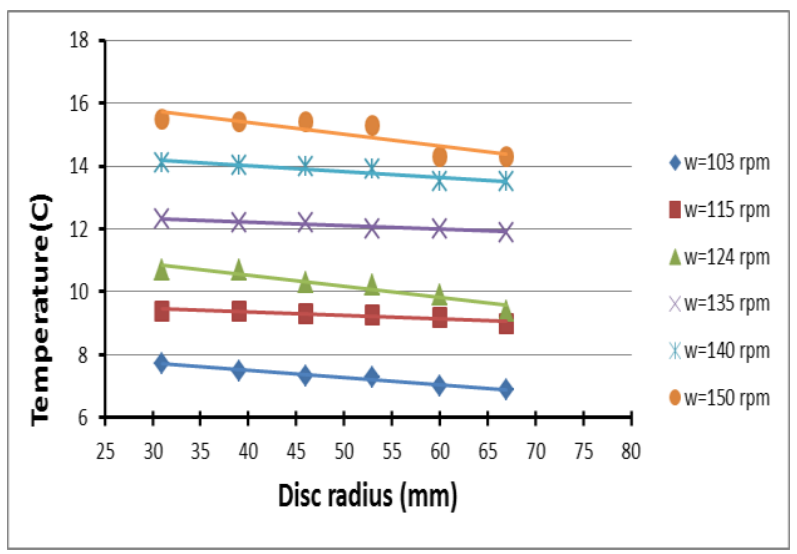

Fig. (10) Disc surface temperature variation versus disc radius at a constant cooling water temperature $\left(T_{\text {cool }}=5^{\circ} \mathrm{C}\right)$ and evaporation power $=385 \mathrm{~W}$

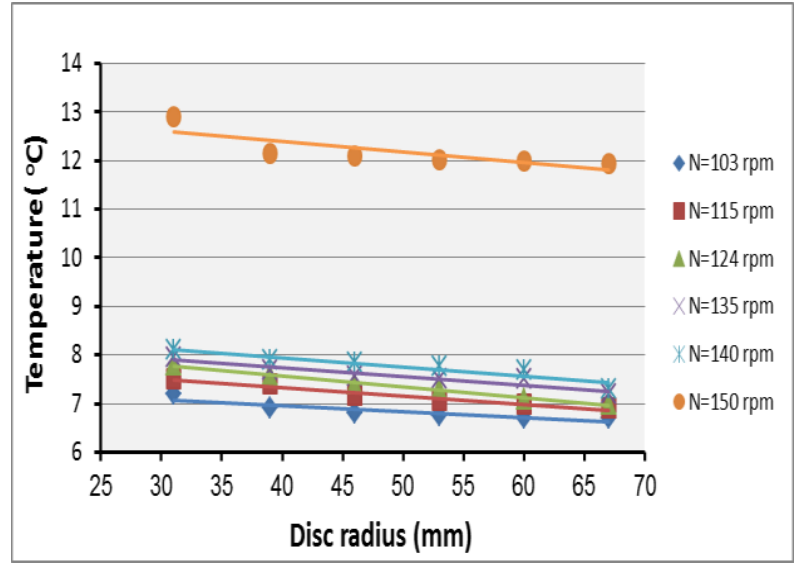

Fig. (11) Disc surface temperature variation versus disc radius at a constant cooling water temperature $\left(\mathrm{T}_{\text {cool }}=5^{\circ} \mathrm{C}\right)$ and evaporation power $=270 \mathrm{w}$

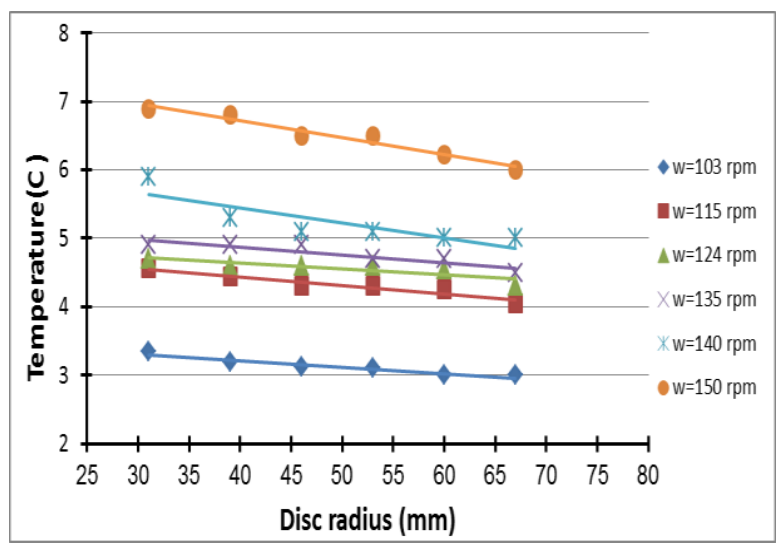

Fig. (12) Disc surface temperature variation versus disc radius at a constant cooling water temperature $\left(T_{\text {cool }}=5^{\circ} \mathrm{C}\right)$ and evaporation power $=168 \mathrm{w}$

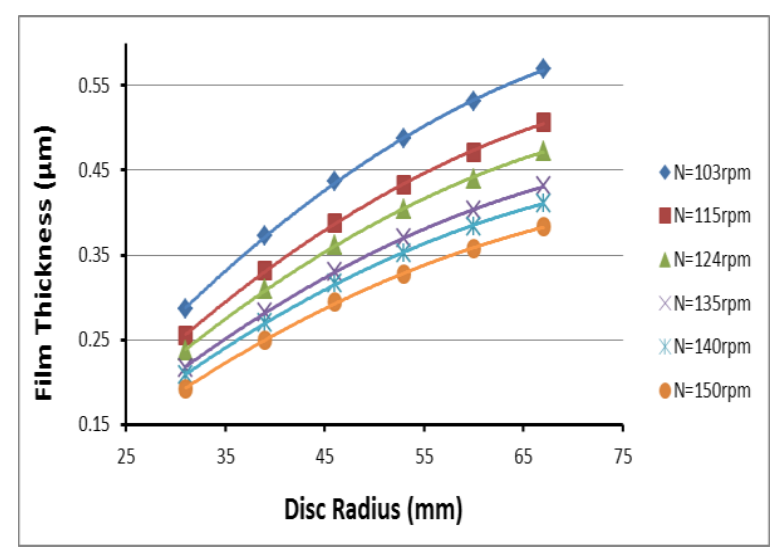

Fig. (13) Film Thickness Distribution along the Radius of rotating disc at $T_{\text {cool }}=5^{\circ} \mathrm{C}$ and $P_{\text {eva. }}=385 \mathrm{~W}$ 


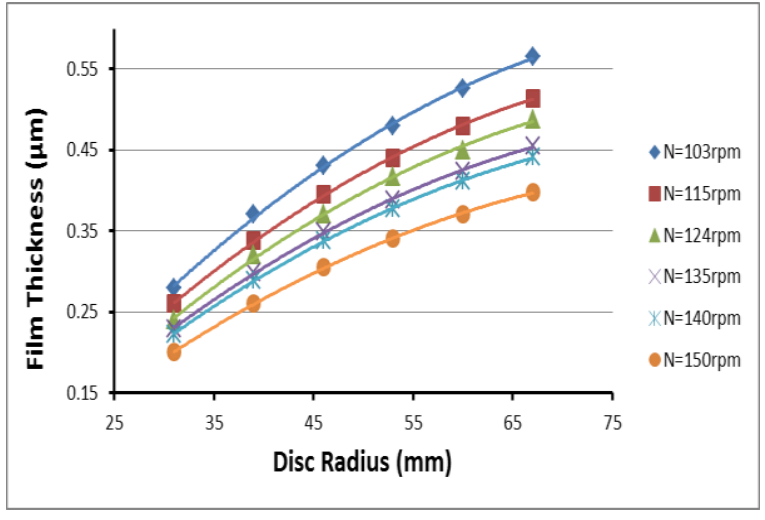

Fig. (14) Film Thickness Distribution along the Radius of rotating disc at $T_{\text {cool }}=5^{\circ} \mathrm{C}$ and $P_{\text {eva. }}=270 \mathrm{~W}$

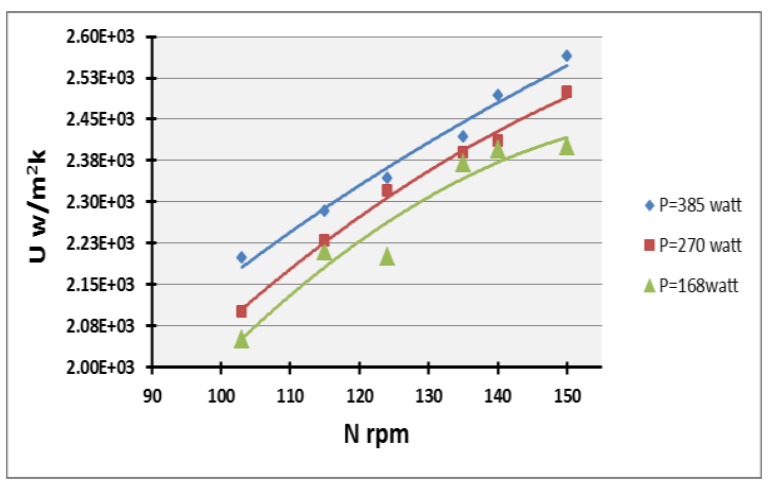

Fig. (15) Overall Heat Transfer Coefficient of the condensing film versus rotational speed at constant $T_{\text {cool }}=5^{\circ} \mathrm{C}$

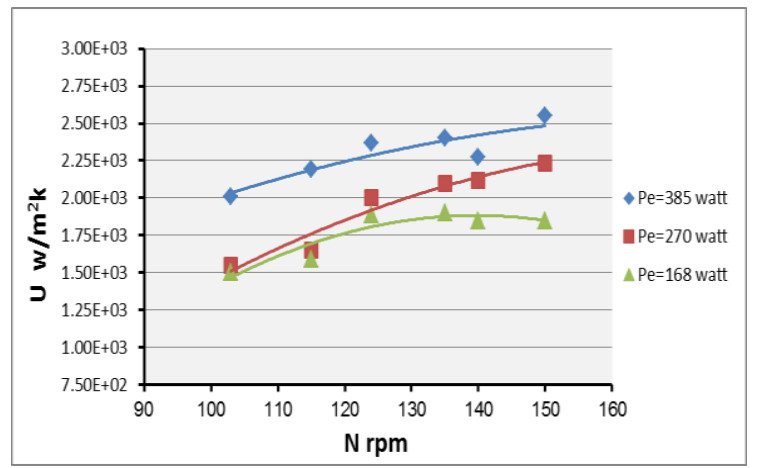

Fig. (16) Overall Heat Transfer Coefficient of the condensing film versus rotational speed at constant $T_{\text {cool }}=5^{\circ} \mathrm{C}$

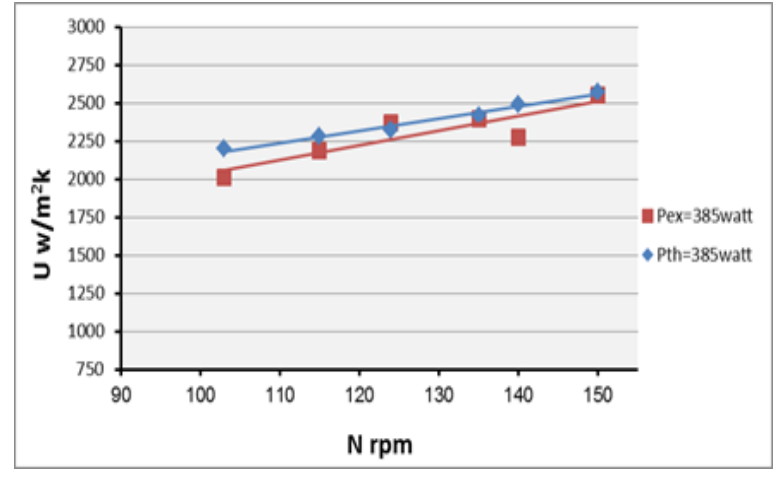

Fig. (5-17): Comparison of overall heat transfer coefficient versus disc rotation speed at constant $T_{\text {cool }}=5^{\circ} \mathrm{C}$

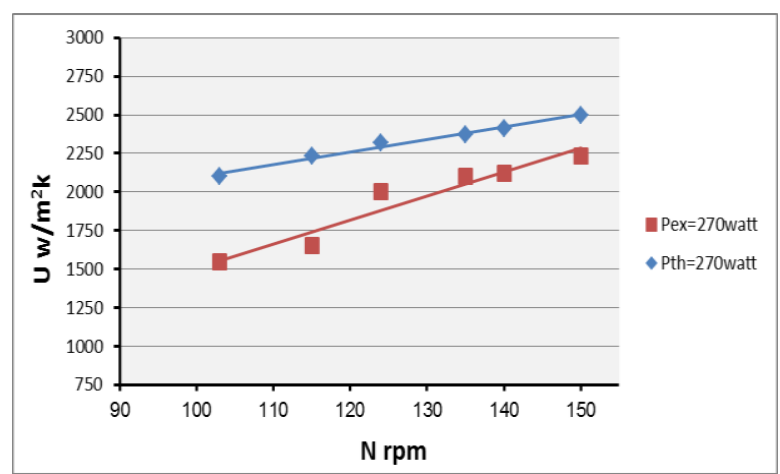

Fig. (18): Comparison of overall heat transfer coefficient versus disc rotation speed at constant $T_{\text {cool }}=5^{\circ} \mathrm{C}$

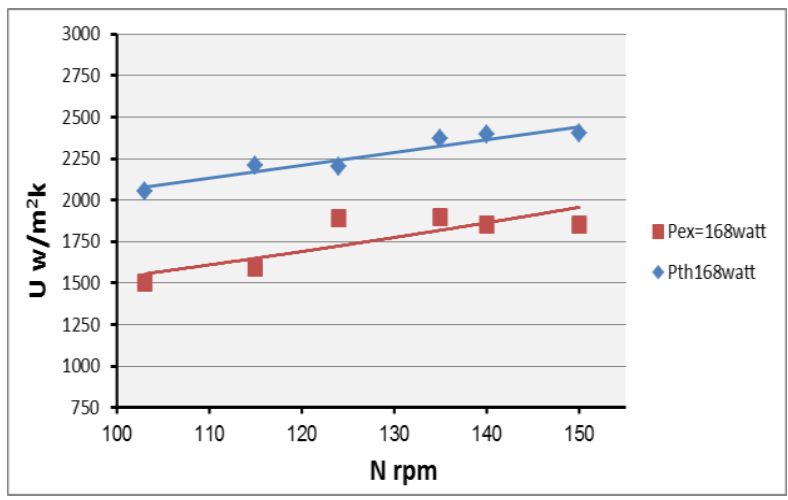

Fig. (19): Comparison of overall heat transfer coefficient versus disc rotation speed at constant $T_{\text {cool }}=5^{\circ} \mathrm{C}$ 